\title{
Throughput of FAST TCP in Asymmetric Networks
}

Fei Ge, Liansheng Tan, and Moshe Zukerman

\begin{abstract}
This letter proposes a model of FAST TCP that captures its dynamics in asymmetric networks. It is found that, as in symmetric networks FAST TCP can achieve stability but its throughput degrades as a function of a measure of the asymmetry that we call asymmetry factor. The theoretical results are validated by simulations.
\end{abstract}

Index Terms-Congestion control, FAST TCP, asymmetric network, throughput.

\section{INTRODUCTION}

$\mathbf{T}$ HE congestion control algorithms applied in the current Internet have avoided severe network congestion while the Internet has grown to its present size. However it is well-known that these algorithms do not scale well with the increase of the bandwidth delay product. This is especially important given the expected increase in Internet transmission capacity. To overcome this key problem, the FAST TCP protocol has been proposed [1], [2], [3], [4]. The performance of FAST TCP has been extensively studied [1], [2], [3], [4], [5], [6]. Although these performance studies indicate that FAST TCP is indeed an important candidate for the future Internet, they did not consider the effect of congestion at the backward, i.e. acknowledgement (ACK), channel. This effect has been shown, mainly by simulations, in [10], [11], [13], [14] to cause instability and reduce throughput for the cases of TCPs Tahoe, Reno and Vegas. Considering these simulation studies, this paper analyses the performance of FAST TCP in an asymmetric network [10], [12], [13] environment. An asymmetric network is characterized by having asymmetric links where there is a significant difference between the capacities available in the two opposite directions of the link. Asymmetric links are prevalent in satellite and access technologies [10]. In such asymmetric networks, it is common to have a situation where the performance of a forward flow is degraded due to congestion in the backward direction [12]. This letter provides an analysis of FAST TCP throughput in asymmetric networks, as a function of a measure which we call Asymmetry Factor that indicates by how much the network is asymmetric. Furthermore, following studies of local and global

Manuscript received October 3, 2007. The associate editor coordinating the review of this letter and approving it for publication was F. Granelli. This research is partially supported by the Program NCET-05-0673 from the Chinese Ministry of Education, and partially by the Australian Research Council (ARC).

F. Ge is with the Dept. of Computer Sci., Central China Normal University, Wuhan 430079, PR China (e-mail: feige@mail.ccnu.edu.cn).

L. Tan is now with The Research School of Information Sciences and Engineering, The Australian National University, Canberra ACT 0200, Australia (e-mail: liansheng.tan@anu.edu.au), on leave from the Dept. of Computer Sci. in Central China Normal University, Wuhan, China.

M. Zukerman is with the ARC Special Research Centre for UltraBroadband Information Networks, EEE Dept., The University of Melbourne, Victoria 3010, Australia (e-mail: mzu@unimelb.edu.au).

Digital Object Identifier 10.1109/LCOMM.2008.071623.

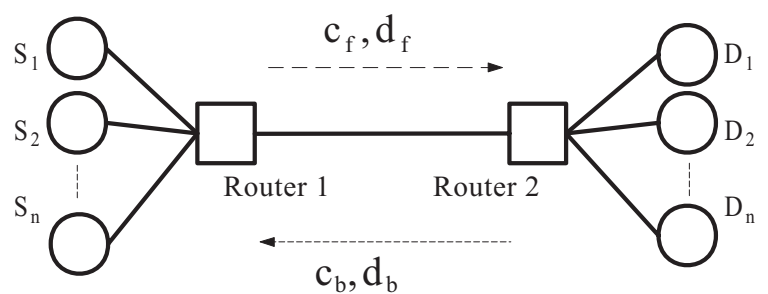

Fig. 1. The single asymmetric link network.

stability of FAST TCP in the absence of feedback queueing delay in [1], [5], [6], here we study the effect of asymmetry on stability.

\section{THE MODEL}

We consider a single asymmetric bottleneck link carrying multiple flows as shown in Fig. 1. Henceforth, the subscript or superscript $f$ and $b$ designate measures related to the forward and backwards links, respectively. The link has a finite forward capacity $c_{f}$ [bytes/s], a forward propagation delay $d_{f}$ and is assumed to have infinite forward buffering storage. The link also has a finite backward capacity $c_{b}$ [bytes/s] (different from $c_{f}$ ), a backward propagation delay $d_{b}$ (different from $d_{f}$ ) and is assumed to have infinite forward buffering storage. Define asymmetry capacity ratio (ACR) of links as $k_{c}=c_{f} / c_{b}$. Due to the different size of forward data packet and ACK packet, the queueing delay in the two directions is not the same. We define asymmetry packet ratio (APR) as $k_{P}=P_{b} / P_{f}$, where $P_{f}$ is a packet size [bytes] from source to link, and $P_{b}$ is the size of a backward packet (ACK packet) [bytes] in the reverse path from link to source. Define asymmetry factor (AF) of a connection in an asymmetric link as

$$
k_{f}=k_{c} \cdot k_{P}=\frac{c_{f} \cdot P_{b}}{c_{b} \cdot P_{f}},
$$

which is the ratio of the transmission time of packets on the bottleneck link on the forward path to that of ACKs on the bottleneck link on the reverse path. As TCP ACKs are much shorter than data packets, $k_{P}$ is much smaller than one [11].

A FAST TCP source updates periodically its congestion window $w$ based on its average Round-Trip Time (RTT) and its estimated queueing delay. Considering the continuous transfer model of [2], we have

$$
\dot{w}(t)=\gamma\left(\frac{d w(t)}{d+q(t)}+\alpha-w(t)\right),
$$

where $w(t)$ is the source congestion window, $\gamma \in(0,1]$, and $\alpha$ is a constant. The send rate [bytes/s] of source $i$ at time $t$ is obtained by

$$
x_{i}^{f}(t)=\frac{w_{i}(t) \cdot P_{i}^{f}}{d+q_{i}^{f}(t)},
$$


so the aggregate rate in the forward and backward links are:

$$
\begin{aligned}
y^{f}(t) & =\sum_{i} x_{i}^{f}\left(t-\tau_{i}^{f}\right), \\
y^{b}(t) & =\sum_{i} x_{i}^{b}\left(t-\tau_{i}^{b}\right) .
\end{aligned}
$$

Considering also ACKs in flight in the backward link, the source $i$ packet rate in the backward direction is given by

$$
x_{i}^{b}(t)=\frac{P_{i}^{b}}{P_{i}^{f}} x_{i}^{f}\left(t-\tau_{i}^{f}\right)=k_{P i} x_{i}^{f}\left(t-\tau_{i}^{f}\right) .
$$

The source $i$ end-to-end queueing delay, $q_{i}(t)$, includes the forward queue delay and the backward delay. Thus,

$$
q_{i}(t)=p^{f}\left(t-\tau_{i}^{b}\right)+p^{b}(t) .
$$

Considering a continuous-time model, as in [7], the queueing delay in the forward direction can be evaluated by

$$
\dot{p}^{f}(t)=\frac{1}{c_{f}}\left(y^{f}(t)-c_{f}\right) .
$$

Accordingly, the queueing delay in the backward direction is evaluated by

$$
\dot{p}^{b}(t)=\frac{1}{c_{b}}\left(y^{b}(t)-c_{b}\right) .
$$

By differentiating (7), we arrive at

$$
\dot{q}_{i}(t)=\dot{p}^{f}\left(t-\tau_{i}^{b}\right)+\dot{p}^{b}(t) .
$$

Substituting (8) and (9) into (10) yields

$$
\dot{q}_{i}(t)=\frac{1}{c_{f}}\left(y^{f}\left(t-\tau_{i}^{b}\right)-c_{f}\right)+\frac{1}{c_{b}}\left(y^{b}(t)-c_{b}\right) .
$$

Substituting (4) and (5) into (11) yields

$\dot{q}_{i}(t)=\frac{1}{c_{f}}\left(\sum_{i} x_{i}^{f}\left(t-\tau_{i}^{f}-\tau_{i}^{b}\right)-c_{f}\right)+\frac{1}{c_{b}}\left(\sum_{i} x_{i}^{b}\left(t-\tau_{i}^{b}\right)-c_{b}\right)$.

Substituting (6) into (12) yields

$$
\begin{gathered}
\dot{q}_{i}(t)=\frac{1}{c_{f}}\left(\sum_{i} x_{i}^{f}\left(t-\tau_{i}^{f}-\tau_{i}^{b}\right)-c_{f}\right) \\
+\frac{1}{c_{b}}\left(\sum_{i} \frac{P_{i}^{b}}{P_{i}^{f}} x_{i}^{f}\left(t-\tau_{i}^{f}-\tau_{i}^{b}\right)-c_{b}\right) .
\end{gathered}
$$

Assuming that $P_{i}^{f}$ and $P_{i}^{b}$ are fixed for all flows, henceforth, they will be denoted by $P_{f}$ and $P_{b}$, respectively. Therefore, we have

$$
\begin{array}{r}
\dot{q}_{i}(t)=\frac{1}{c_{f}}\left(\sum_{i} x_{i}^{f}\left(t-\tau_{i}^{f}-\tau_{i}^{b}\right)-c_{f}\right) \\
+\frac{c_{f}}{c_{b}} \frac{P_{b}}{P_{f}}\left(\frac{1}{c_{f}}\left(\sum_{i} x_{i}^{f}\left(t-\tau_{i}^{f}-\tau_{i}^{b}\right)-\frac{P_{f}}{P_{b}} c_{b}\right)\right) \\
=\frac{1}{c_{f}}\left(\sum_{i} x_{i}^{f}\left(t-\tau_{i}^{f}-\tau_{i}^{b}\right)-c_{f}\right) \\
\left.+k_{f} c_{f}\left(\sum_{i} x_{i}^{f}\left(t-\tau_{i}^{f}-\tau_{i}^{b}\right)-\frac{1}{k_{f}} c_{f}\right)\right) \\
=\left(1+k_{f}\right) \frac{1}{c_{f}}\left(\sum_{i} x_{i}^{f}\left(t-\tau_{i}^{f}-\tau_{i}^{b}\right)-\frac{2 c_{f}}{1+k_{f}}\right) .
\end{array}
$$

Assuming $\tau_{i}^{f}$ and $\tau_{i}^{b}$ to be the same for all flows, henceforth, they will be denoted by $\tau^{f}$ and $\tau^{b}$, respectively. Substituting (3) into (14) yields

$$
\dot{q}_{i}(t)=\left(1+k_{f}\right) \frac{1}{c_{f}}\left(\sum_{i} \frac{w\left(t-\tau_{i}^{f}-\tau_{i}^{b}\right) \cdot P_{f}}{d+q\left(t-\tau_{i}^{f}-\tau_{i}^{b}\right)}-\frac{2 c_{f}}{1+k_{f}}\right) .
$$

Linearizing (2) and (15), at the equilibrium point $\left(q_{0}, w_{0}\right)$, where $x_{0}=\alpha / q_{0}, R_{0}=d+q_{0}=\tau^{f}+\tau^{b}, w_{0}=\left(d+q_{0}\right) x_{0}$, $x_{0}=c_{f} / n$, we obtain

$$
\begin{gathered}
\Delta \dot{q}(t)=\left(1+k_{f}\right) \frac{n P_{f}}{c_{f}}\left(\frac{1}{R_{0}} \Delta w\left(t-R_{0}\right)-\frac{w_{0}}{R_{0}^{2}} \Delta q\left(t-R_{0}\right)\right), \\
\Delta \dot{w}(t)=-\frac{\gamma q_{0}}{R_{0}} \Delta w(t)-\frac{w_{0} d}{R_{0}^{2}} \Delta q(t) .
\end{gathered}
$$

Taking the Laplace transform of (16), (17) and after some algebra, we obtain

$$
\begin{gathered}
s Q(s)=\left(1+k_{f}\right) \frac{n P_{f}}{c_{f}}\left(\frac{1}{R_{0}} W(s) e^{-R_{0} s}-\frac{w_{0}}{R_{0}^{2}} Q(s) e^{-R_{0} s}\right), \\
s W(s)=-\frac{\gamma q_{0}}{R_{0}} W(s)-\frac{w_{0} d}{R_{0}^{2}} Q(s) .
\end{gathered}
$$

Thus, we can obtain the open loop transfer function from $W(s)$ to $W(s)$ as follows

$$
L(s)=\frac{-\frac{\gamma d}{R_{0}^{2}}\left(1+k_{f}\right) n P_{f} e^{-R_{0} s}}{\left(s+\frac{\left(1+k_{f}\right) n P_{f} e^{-R_{0} s}}{R_{0}}\right)\left(s+\frac{\gamma q_{0}}{R_{0}}\right)} .
$$

Using the exponential function approximation

$$
e^{-R_{0} s}=\frac{1}{1+R_{0} s}
$$

we obtain

$$
L(s)=\frac{-\frac{\gamma d}{R_{0}^{2}}\left(1+k_{f}\right) n P_{f}}{\left(s+R_{0} s^{2}+\frac{\left(1+k_{f}\right) n P_{f}}{R_{0}}\right)\left(s+\frac{\gamma q_{0}}{R_{0}}\right)} .
$$

\section{STABility AND Throughrut}

Let $\hat{x}_{i}^{f}$ denote the sending rate [packets/s] of source $i$ in the forward direction, and $\hat{x}_{i}^{b}$ denote the flow rate in the backward direction. With regard to the throughput of source $i$, which is denoted as $x_{i}$, we have the following theorem.

Theorem 1. The throughput of a FAST TCP source in a single asymmetric link is stable and the value at the equilibrium point is

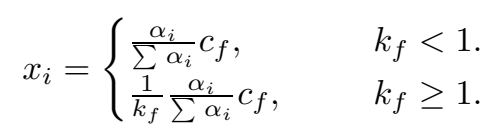

Proof: It is seen that the three poles of (22) are on the left-half plane, so the system is stable in terms of window size. Subsequently, the throughput of a FAST TCP source in a single asymmetric link is stable.

For every sources $i$ in stable status, we obtain $\sum_{i} x_{i}^{f} \leq c_{f}$ and $\sum_{i} x_{i}^{b} \leq c_{b}$, which is $\sum_{i} \hat{x}_{i}^{f} \leq c_{f} / P_{f}$ and $\sum_{i} \hat{x}_{i}^{b} \leq$ $c_{b} / P_{b}$. For every flow self clocking, $\hat{x}_{i}^{f}=\hat{x}_{i}^{b}$. When $k_{f} \leq 1$, $c_{f} / P_{f} \leq c_{b} / P_{b}, \sum_{i} \hat{x}_{i}^{f}(i) \leq c_{f} / P_{f} \leq c_{b} / P_{b}$. At equilibrium point, $\sum_{i} \hat{x}_{i}^{f}=c_{f} / P_{f}$. When $k_{f}>1, c_{f} / P_{f}>c_{b} / P_{b}$, $\sum_{i} \hat{x}_{i}^{b} \leq c_{b} / P_{b} \leq c_{f} / P_{f}$. At equilibrium point, $\sum_{i} \hat{x}_{i}^{b}=$ $c_{b} / P_{b}$. 


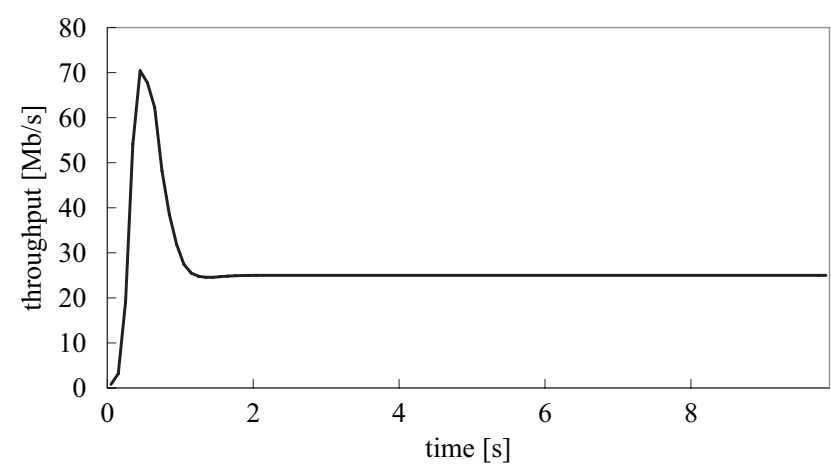

Fig. 2. The throughput when $P_{f}=1000$ bytes and $c_{b}=1 \mathrm{Mb} / \mathrm{s}$.

Fast TCP achieves proportional fairness [4], then $\hat{x}_{i}^{f}=\frac{\alpha_{i}}{\sum \alpha_{i}} \sum \hat{x}_{i}^{f}=\frac{\alpha_{i}}{\sum \alpha_{i}} \frac{c_{f}}{P_{f}}$ when $k_{f} \leq 1$, and $\hat{x}_{i}^{b}=\frac{\alpha_{i}}{\sum \alpha_{i}} \sum \hat{x}_{i}^{b}=\frac{\alpha_{i}}{\sum \alpha_{i}} \frac{c_{b}}{P_{b}}$ when $k_{f}>1$.

Because a source throughput $x(i)$ is the product of source sending rate $\hat{x}_{i}^{f}$ and the forward data packet size, the following can be obtained

$$
x_{i}= \begin{cases}\hat{x}_{i}^{f} P_{f}=\frac{\alpha_{i}}{\sum \sum \alpha_{i}} c_{f}, & k_{f}<1, \\ \hat{x}_{i}^{b} P_{f}=\frac{1}{k_{f}} \frac{\alpha_{i}}{\sum \alpha_{i}} c_{f}, & k_{f} \geq 1 .\end{cases}
$$

Throughput improvement can be achieved by increasing $P_{f}$ in single asymmetric network when $k_{f}>1$.

\section{Simulation Results}

In this section we demonstrate the asymmetry effect on the stability and throughput of FAST TCP at equilibrium using NS2 [8], [9]. To simplify, we mainly investigate the effect of backward capacity when $P_{f}, P_{b}, c_{f}$ are set fixed with a single source. Consider a single asymmetric link network with the following setting: $c_{f}=100 \mathrm{Mb} / \mathrm{s}, P_{f}=1000$ bytes, $P_{b}=40$ bytes, $d_{f}=20 \mathrm{~ms}, d_{b}=20 \mathrm{~ms}, \alpha=1000$. The values presented are the average throughput measured in every consecutive $0.1 \mathrm{~s}$ interval. When $c_{b}=1 \mathrm{Mb} / \mathrm{s}$, we obtain $k_{f}=4$, at equilibrium point $x_{0}=\frac{c_{f}}{k_{f}}=25 \mathrm{Mb} / \mathrm{s}$, $d / q_{0}=d \cdot x_{0} / \alpha=0.125<1$. The result is shown in Fig. 2. From Fig. 2 we observe that FAST TCP source is stable and its throughput satisfies Theorem 1 . When $c_{b}=0.1 \mathrm{Mb} / \mathrm{s}$, the stability and throughput results are shown in Fig. 3. The results are consistent with Theorem 1. Fig. 4 shows the throughput results when the packet size is 2000 bytes (only for simulation) and $c_{b}=0.1 \mathrm{Mb} / \mathrm{s}$. Comparing with a packet size of 1000 bytes, the throughput is doubled.

\section{CONCLUSion}

We have analyzed the stability and throughput of FAST TCP for a network with a single asymmetric bottleneck link. Our analysis based on a continuous transfer model, which considers forward queueing delay and backward queueing delay for FAST TCP, has shown that asymmetry does not affect stability. However, the forward throughput depends on the asymmetry factor. The results have been validated by simulations.

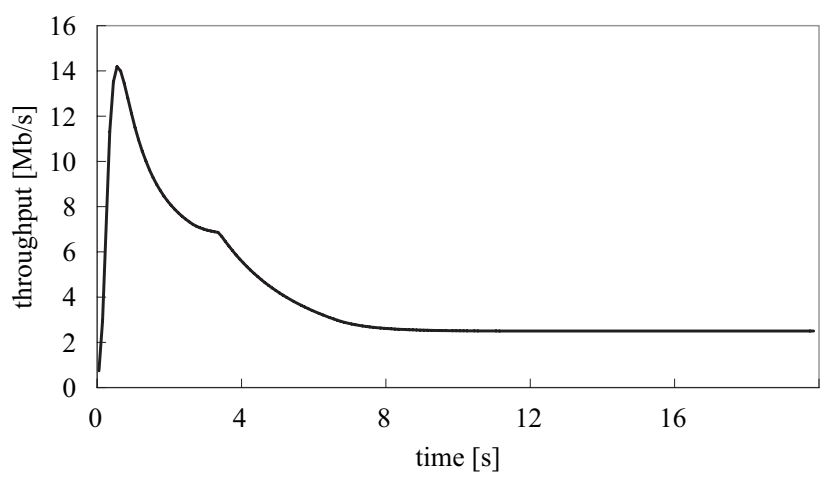

Fig. 3. The throughput when $P_{f}=1000$ bytes and $c_{b}=0.1 \mathrm{Mb} / \mathrm{s}$.

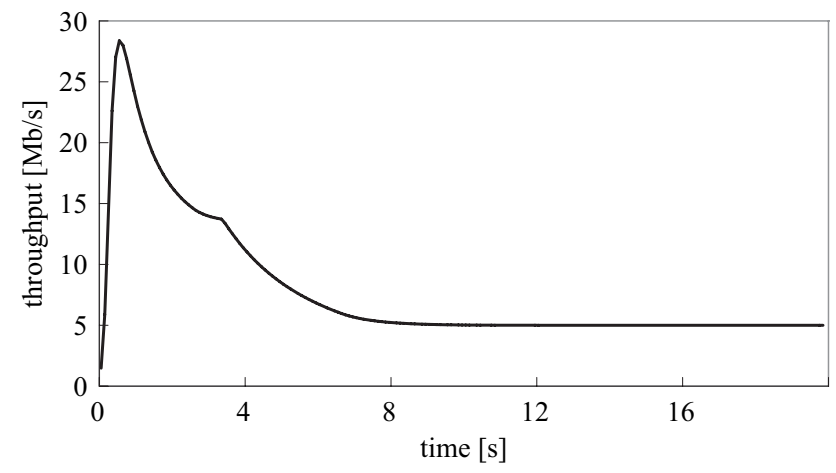

Fig. 4. The throughput when $P_{f}=2000$ bytes and $c_{b}=0.1 \mathrm{Mb} / \mathrm{s}$.

\section{REFERENCES}

[1] J. Y. Choi, K. Koo, J. S. Lee, and S. H. Low, "Global stability of FAST TCP in single-link single-source network," in Proc. 44th IEEE Conference on Decision and Control, and the European Control Conference 2005, Seville, Spain, Dec. 2005.

[2] C. Jin, D. Wei, and S. H. Low, "FAST TCP: motivation, architecture, algorithms, performance," in Proc. IEEE INFOCOM 2004, Hong Kong, Mar. 2004.

[3] C. Jin, D. Wei, and S. H. Low, "FAST TCP for high-speed long-distance networks," Internet draft draft-jwl-tcp-fast-01.txt. [Online]. Available: http://netlab.caltech.edu/pub/papers/draft-jwl-tcp-fast-01.txt.

[4] C. Jin et al., "FAST TCP: from theory to experiments," IEEE Network, vol. 19, no. 1, pp. 4-11, Jan./Feb. 2005.

[5] L. Tan, C. Yuan, and M. Zukerman, "FAST TCP: fairness and queuing issues," IEEE Commun. Lett., vol. 9, no. 8, pp. 762-764, Aug. 2005.

[6] J. Wang, A. Tang, and S. H. Low, "Local stability of FAST TCP," in Proc. IEEE Conference on Decision and Control (CDC), Paradise Island, Bahamas, Dec. 2004.

[7] J. Wang, D. X. Wei, and S. H. Low, "Modeling and stability of FAST TCP," in Proc. IEEE INFOCOM 2005, Miami, FL, Mar. 2005.

[8] The network simulator: ns-2. [Online]. Available: http://www.isi.edu/ nanam $/ \mathrm{ns} /$

[9] T. Cui and L. L. H. Andrew, "FAST TCP simulator module for ns-2, version 1.1.” [Online]. Available: http://www.cubinlab.ee.mu.oz.au/ ns 2 fasttcp.

[10] C. P. Fu and S. C. Liew, "A remedy for performance degradation of TCP vagas in asymmetric networks," IEEE Commun. Lett., vol. 7, no. 8, pp. 42-44, Jan. 2003.

[11] T. V. Lakshman, U. Madhow, and B. Suter, "TCP/IP performance with random loss and bidirectional congestion," IEEE/ACM Trans. Networking, vol. 8 , no. 5, pp. 541-555, Oct. 2000.

[12] F. Ge and L. Tan, "Improving FAST TCP performance in asymmetric networks," in Proc. IEEE 2nd International Conference on Wireless Broadband and Ultra Wideband Communications (AusWireless'07), Sydney, Australia, Aug. 2007.

[13] H. Balakrishnan, V. N. Padmanabhan, G. Fairhurst, and M. Sooriyabandara, "TCP performance implications of network path asymmetry," rfc3449, http://www.ietf.org/rfc.html, Dec. 2002.

[14] J. Sun, S. Chan, K. T. Ko, G. Chen, and M. Zukerman, "Instability effects of two-way traffic in a TCP/AQM system," Computer Commun., vol. 30, no. 10, pp. 2172-2179, July 2007. 